\title{
Relación entre la Ansiedad Social y las Dimensiones de la Personalidad en Adolescentes Españoles
}

\author{
Relationship between Social Anxiety and Personality Dimensions in Spanish \\ Adolescents
}

\author{
Beatriz Delgado ${ }^{1}$, Cándido J. Inglés ${ }^{2}$, David Aparisi ${ }^{1}$, José M. García-Fernández ${ }^{1}$ y María C. \\ Martínez-Monteagudo ${ }^{1}$
}

\begin{abstract}
Resumen
El objetivo de este estudio fue analizar la relación entre la ansiedad social y las variables de personalidad (neuroticismo, extraversión y psicoticismo) en una muestra de 2022 (51.1\% varones) adolescentes españoles de 12 a 16 años. La ansiedad social se evaluó utilizando el cuestionario Social Phobia and Anxiety Inventory y para el análisis de las variables de personalidad se administró el Cuestionario de Personalidad de Eysenck. Los resultados indican que los estudiantes con alta ansiedad social presentan un perfil de personalidad compuesto por altas puntuaciones en neuroticismo, bajas puntuaciones en extraversión y niveles similares en psicoticismo. Los resultados de la regresión logística señalan que los estudiantes con alta ansiedad social tienen una mayor probabilidad de obtener altas puntuaciones en neuroticismo y menor probabilidad de presentar un rasgo de personalidad extravertido. Además, la baja extraversión y el alto neuroticismo son predictores positivos de la ansiedad social.
\end{abstract}

Palabras clave: adolescencia, ansiedad social, personalidad, educación secundaria

\begin{abstract}
The aim of this study was to analyze the relationship between social anxiety and personality variables (neuroticism, extraversion and psychoticism) on a sample of 2022 (51.1\% male) Spanish adolescents aged 12 to 16 years. Social anxiety was assessed using the questionnaire Social Phobia and Anxiety Inventory and for the analysis of personality variables the Eysenck Personality Questionnaire was administered. The results indicate that students with high social anxiety have a personality profile consisting of high scores in neuroticism or emotional instability, low scores on extraversion and psychoticism similar levels. The results of logistic regression indicate that students with high social anxiety are more likely to get high scores on neuroticism and less likely to have an extroverted personality trait. In addition, low extraversion and high neuroticism are positive predictors of social anxiety.
\end{abstract}

Keywords: adolescence, social anxiety, personality, secondary education

Este artículo de investigación ha sido financiado a través del Proyecto SEJ 2004-07311/EDUC perteneciente al Plan Nacional de Investigación Científica, Desarrollo e Innovación Tecnológica del MEC concedido al segundo autor.

\footnotetext{
${ }^{1}$ Departamento de Psicología Evolutiva y Didáctica. Universidad de Alicante, España.

${ }^{2}$ Departamento de Psicología de la Salud. Universidad Miguel Hernández de Elche, España.

Correspondencia: Dra. Beatriz Delgado. Departamento de Psicología Evolutiva y Didáctica. Universidad de Alicante. Ap. Correos 99, 03080. Alicante, España. Correo: beatriz.delgado@ua.es
} 


\section{Introducción}

La ansiedad social en la infancia y adolescencia resulta un problema grave para el desarrollo normal del menor. Los niños y adolescentes con esta problemática presentan niveles elevados de malestar emocional y una interferencia considerable en su funcionamiento social y académico (Gren-Landell et al., 2009), especialmente durante las interacciones sociales con los compañeros de clase y con personas de su misma edad (Blöte, Miers, Heyne, \& Westenberg, 2015; Inglés, Delgado, García-Fernández, RuizEsteban, \& Díaz-Herrero, 2010).

También han sido detectadas algunas repercusiones de la ansiedad social sobre el funcionamiento escolar. Así, los estudiantes con alta ansiedad social demuestran ciertas dificultades académicas y un menor rendimiento académico (Delgado, Inglés, \& García-Fernández, 2014) que los estudiantes sin alta ansiedad social. Además, los estudiantes con ansiedad social participan en menos actividades extraescolares, sienten más estrés por las tareas académicas y presentan más absentismo escolar (Van Roy, Kristensen, Groholt, \& Clench-Aas, 2009).

Numerosas razones justifican el estudio de la ansiedad social en la adolescencia. En primer lugar, aparece frecuentemente a edades tempranas del desarrollo y su curso suele ser crónico si no es tratado, lo que puede generar una interferencia considerable sobre el funcionamiento y desarrollo social de los menores. Además, el malestar producido por la ansiedad social y sus respuestas evitativas repercuten negativamente en diferentes áreas funcionales de los niños. Así, la escasa participación en clase, la resistencia a presentar trabajos en el aula o la tendencia a evitar preguntar al profesor (Bernstein, Bernat, Davis, \& Layne, 2008), puede contribuir que el estudiante obtenga calificaciones académicas más bajas y fracaso escolar (Delgado et al., 2014) y mantenga un pobre autoconcepto académico (Delgado, Inglés, \& García-Fernández, 2013). Igualmente, la evitación de las relaciones con los compañeros genera aislamiento y una baja aceptación en el grupo de iguales (Inglés et al., 2010).
Ansiedad social $y$ personalidad en la adolescencia

A pesar de existir una amplia evidencia empírica que señala el inicio de la ansiedad social durante la infancia o adolescencia (Bados, 2001), son escasos los estudios que analizan las características de personalidad de los ansiosos sociales en edades tempranas (Zhou, Xu, Inglés, Hidalgo, \& La Greca, 2008).

Los factores de personalidad que subyacen a la ansiedad social han sido evaluados mediante estudios correlacionales y predictivos con muestras clínicas (Beidel et al., 2007) y comunitarias (Calvete, Orue, \& Gamez-Guadix, 2016; Mulder \& Aken, 2014; Zhao, Zhang, Chen, \& Zhou, 2014; Zhou et al., 2008). La evidencia empírica coincide en afirmar que la baja extraversión y el alto neuroticismo o inestabilidad emocional están asociados con la ansiedad social (Inglés, Hidalgo, \& Méndez, 2005), mientras que la relación con el rasgo de psicoticismo resulta muy débil (Darwill et al., 1992). A continuación, se detallan los principales resultados extraídos de la revisión de estudios sobre ansiedad social, neuroticismo y extraversión.

El neuroticismo consiste en la tendencia a experimentar emociones negativas como miedo, tristeza, vergüenza, ira y culpa. El neuroticismo incluye mayor susceptibilidad al malestar psicológico $\mathrm{y}$, por ende, a presentar ideas irracionales, menos control de impulsos y estrategias de afrontamiento ante el estrés (Ehrler, Evans, \& McGhee, 1999).

Los niños con un marcado rasgo de neuroticismo suelen manifestar mayores problemas de ansiedad (Woodward \& Ferguson, 2001), de relación social y mayores niveles de inhibición conductual (Slobodskaya, 2007). Así, Ehrler et al. (1999) obtuvieron, a través de un estudio correlacional con NEO Personality Inventory-Revised (NEO PI-R; Costa \& McCrae, 1999) en una muestra comunitaria compuesta por 83 escolares de 9 a13 años, que las puntuaciones de neuroticismo se relacionaban negativa y significativamente con la ansiedad y los problemas de interacción social.

Utilizando las mismas dimensiones del modelo de cinco factores a través del Inventory of Child Individual Differences (ICID; Halverson et al., 2003) y en una muestra más amplia ( $n=1013$; 
rango de edad: 3-18 años), Slobodskaya (2007) encontró una alta relación entre neuroticismo autoinformado y problemas de relación con los compañeros.

Por su parte, Woodward y Ferguson (2001) examinaron la tasa de neuroticismo (Eysenck Personality Inventory, EPI; Eysenck \& Eysenck, 1964) que existía en los sujetos con trastornos de ansiedad (entre los cuales había un $2.9 \%$ de ansiosos sociales) extraído de muestra de 964 neozelandeses de 14 años. Los resultados indicaron que el $78.3 \%$ de los adolescentes con tres trastornos de ansiedad, $49.2 \%$ de adolescentes con dos trastornos de ansiedad y $35.1 \%$ de adolescentes con un trastorno de ansiedad informaban de puntuaciones elevadas de neuroticismo (cuartil superior). Ese porcentaje disminuía a $5.2 \%$ en un grupo de estudiantes sin ningún trastorno de ansiedad.

Ese mismo año, Inglés et al. (2001) analizaron entre otras variables, la relación entre fobia social y rasgos de personalidad, estos últimos evaluados a través de dos versiones del autoinforme Eysenck Personality Questionnaire (EPQ-J y EPQ-A; Eysenck \& Eysenck, 1975). La muestra de estudio estuvo compuesta por 538 adolescentes españoles. Los hallazgos evidenciaron el poder explicativo del neuroticismo sobre la ansiedad social. Igualmente, encontraron que la proporción de adolescentes inestables emocionales (alto neuroticismo) con fobia social fue significativamente mayor que la de los estables emocionales (bajo neuroticismo). Además, aquellos sujetos con altas puntuaciones en neuroticismo presentaban mayores dificultades para relacionarse con personas del sexo opuesto, manifestaban más problemas para hablar en público y mostrar conductas asertivas.

Con el propósito de evaluar la validez de constructo de la Social Anxiety Scale for Adolescents (SAS-A; La Greca, 1998), Zhou et al. (2008) analizaron la correlación entre este instrumento y sus subescalas con el International Personality Item Pool (IPIP; Goldberg, 1999) en una muestra compuesta por 296 estudiantes chinos de educación secundaria. Los resultados revelaron una relación positiva y significativa entre las puntuaciones de neuroticismo y las puntuaciones totales del instrumento, y sus subescalas de miedo a la evaluación negativa, malestar y evitación social en situaciones novedosas y en situaciones sociales generales. Estos hallazgos fueron hallados recientemente en otro estudio correlacional utilizando el NEO-Five-Factor Inventory (NEO-FFI; Costa \& McCrae, 1999) en una muestra de estudiantes chinos de 11 a 19 años (Zhao et al. 2014).

Por su parte, Mulder y Aken (2014) encontraron, en una muestra de 1814 adolescentes holandeses, que el neuroticismo (evaluado a través del Big Five Inventory, BFI; John \& Srivastava, 1999) correlacionaba positiva y con magnitud alta con la ansiedad social, y estas variables a su vez, predecían el nivel de victimización o acoso en los menores.

Recientemente, en un estudio longitudinal realizado con 1440 adolescentes españoles se analizó la asociación entre los rasgos de personalidad (Big Five Questionnaire-Children, BFQ-C; Barbaranelli, Caprara, \& Rabasca, 1998), la ansiedad social, la depresión y la acoso entre iguales (Calvete et al., 2016). Se halló que el neuroticismo se relacionaba positivamente con la ansiedad social y la depresión en tres momentos temporales, y era uno de los rasgos de personalidad que más predecía ambos trastornos.

En esta línea, el neuroticismo ha sido identificado como factor altamente relacionado con la ansiedad social también en muestras comunitarias de jóvenes (Merikangas, Avenenoli, Acharyya, Zhang, \& Angst, 2002), universitarios (Norton et al., 1997) y en población clínica adolescente (Beidel et al., 2007).

Si el neuroticismo aparecía como factor de riesgo para problemas de relación y ansiedad, la alta extraversión ha sido considerada como un elemento protector de estas dificultades en la infancia y adolescencia (Ehrler et al., 1999; Inglés et al., 2001; Slobodskaya, 2007, Woodward \& Ferguson, 2001). De este modo, los estudios correlacionales descritos anteriormente, obtuvieron una moderada y significativa relación negativa entre la extraversión y los problemas de ansiedad y problemas de relación con los iguales (Ehrler et al., 1999; Slobodskaya, 2007), y extraversión y ansiedad social (Mulder \& Aken, 2014; Zhao et al., 2014; Zhou et al., 2008). Además, Slobodskaya (2007) indicó que los niños y adolescentes extravertidos tenían menos riesgo 
de manifestar problemas cuando se relacionaban con niños de su edad.

En esta misma línea, el estudio de Inglés et al. (2001) reveló el poder explicativo de la introversión (o baja extraversión) sobre la ansiedad social. Igualmente, hallaron que la proporción de adolescentes introvertidos (baja extraversión) con fobia social fue significativamente mayor que la de los extravertidos. Además, los sujetos introvertidos presentaban mayores dificultades para relacionarse con los amigos y con personas del sexo opuesto, manifestaban más problemas para hablar en público y mostrar conductas asertivas.

Por su parte, Calvete et al. (2016) encontraron que la ansiedad social era explicada negativa y significativamente por la extraversión y adoptaba un papel amortiguador de los efectos de la ansiedad social sobre el acoso escolar. Así, los adolescentes que presentaban ansiedad social tenían menos probabilidad de ser victimizados cuando sus niveles de extraversión eran altos respecto a los adolescentes ansiosos con baja extraversión.

Estos hallazgos han sido confirmados en los trabajos de Beidel y colaboradores en muestras de adolescentes diagnosticados con ansiedad social (Beidel et al., 2007). Respecto al grupo control, los ansiosos sociales informaban de puntuaciones medias significativamente menores en la escala de Extraversión del EPI y del EPQ-J.

Sin embargo, el poder predictivo de la extraversión no aparece de modo concluyente en algunos estudios. Así, Norton et al. (1997) señalaron que la extraversión sólo explicaba un $3 \%$ de la ansiedad social, porcentaje muy inferior al hallado para el miedo a las respuestas de ansiedad y el neuroticismo.

\section{El presente estudio}

La revisión de la investigación previa relativa a las relaciones entre ansiedad social y la personalidad presenta varias limitaciones. En primer lugar, investigaciones anteriores han estudiado la relación de las variables de personalidad con la ansiedad social pero sin contemplar el rasgo de psicoticismo y la relación predictiva bidireccional entre ambas variables en adolescentes de Educación Secundaria. Asimismo, los estudios previos tampoco han incluido los tamaños del efecto o magnitud de las diferencias y la elaboración de modelos logísticos, lo que imposibilita interpretar la relevancia teórica y práctica de los resultados.

Teniendo en cuenta estas limitaciones, el presente estudio tiene un triple objetivo: a) analizar las diferencias en personalidad entre estudiantes españoles de ESO con alta y baja ansiedad social, b) determinar en qué medida la alta ansiedad social pronostica altas puntuaciones en neuroticismo, extraversión y psicoticismo, y c) examinar el papel explicativo que tienen los rasgos de personalidad sobre la alta ansiedad social.

A partir de los hallazgos de investigaciones previas, se espera que los estudiantes con alta ansiedad social presenten: 1) puntuaciones significativamente mayores en neuroticismo, 2) puntuaciones significativamente menores en extraversión, y 3) puntuaciones similares en psicoticismo. Igualmente a través de los análisis predictivos (regresión logística), se espera que: 4) la alta ansiedad social sea predictor positivo y significativo del neuroticismo y de la baja extraversión, 5) la alta ansiedad social y el psicoticismo no se relacionen significativamente, y 6) los rasgos de personalidad neuroticismo y extraversión sean predictores significativos de la alta ansiedad social.

\section{Método}

\section{Participantes}

Se realizó un muestreo aleatorio por conglomerados, siendo las unidades primarias de muestreo las zonas geográficas (centro, norte, sur, este y oeste) de dos provincias españolas pertenecientes a dos comunidades autónomas. Las unidades secundarias fueron los centros escolares de cada zona geográfica $\mathrm{y}$, finalmente, las unidades terciarias fueron las aulas. Con el fin de que todas las zonas geográficas estuvieran representadas se seleccionaron aleatoriamente 20 centros de áreas rurales y urbanas, 14 públicos y 6 privados. Cada zona geográfica estuvo representada por una media de dos centros. Una vez determinados los centros del estudio, se seleccionaron aleatoriamente cuatro aulas computándose aproximadamente 120 sujetos por centro. 
El total de sujetos seleccionados fue 2267, de los que $116(5.12 \%)$ fueron excluidos por errores $\mathrm{u}$ omisiones en sus respuestas o por no obtener por escrito el consentimiento informado de los padres para participar en la investigación y 129 (5.69\%) fueron excluidos por ser extranjeros con importantes déficits en el dominio de la lengua española.

La muestra final se compuso de 2022 estudiantes (1033 chicos y 989 chicas) de ESO, con un rango de edad de 12 a 16 años $(M=13.81$; $D T=1.35)$. La distribución de la muestra por curso académico fue la siguiente: $1^{\circ}$ ESO (576; 309 chicos y 267 chicas), $2^{\circ}$ ESO $(505 ; 251$ chicos y 254 chicas), $3^{\circ}$ ESO (502; 260 chicos y 242 chicas) y $4^{\circ}$ de $\operatorname{ESO}(439 ; 213$ chicos y 226 chicas). La prueba chi-cuadrado de homogeneidad de la distribución de frecuencias reveló la ausencia de diferencias estadísticamente significativas entre los ocho grupos de Sexo $\mathrm{x}$ Curso $\left(\chi^{2}=3.16 ; p=.37\right)$. La composición étnica de la muestra fue la siguiente: $88.9 \%$ españoles, $6.34 \%$ hispanoamericanos, $3.37 \%$ resto Europa, $0.75 \%$ asiáticos y $0.64 \%$ árabes.

\section{Instrumentos}

\section{Social Phobia and Anxiety Inventory (SPAI; Turner, Beidel, Dancu \& Stanley, 1989)}

La ansiedad social se evaluó utilizando la subescala Fobia Social del cuestionario. El SPAI es una medida de autoinforme compuesta por 32 ítems, diseñada para evaluar sintomatología cognitiva, fisiológica y motora de la ansiedad social en la adolescencia. Cada ítem se puntúa según una escala Likert de siete puntos (1=nunca; $7=$ siempre). El SPAI es una de las herramientas con mejores garantías psicométricas para examinar la ansiedad social en población comunitaria adolescente anglo e hispanoparlante (García-López, De Los Reyes, \& Salvador, 2015). La adaptación española del SPAI fue realizada por Olivares, García-López, Hidalgo, Turner, \& Beidel (1999) a través de la traducción y adecuación de los ítems para población adolescente, obteniendo evidencias de fiabilidad y validez de sus puntuaciones. Los

resultados mostraron coeficientes adecuados de consistencia interna (.95 para la subescala de Fobia Social. Estudios posteriores han encontrado índices satisfactorios de consistencia interna (Inglés et al., 2010) y estabilidad temporal. La subescala Fobia Social del SPAI obtuvo un índice adecuado Alpha de Cronbach (.95) en el presente estudio.

\section{Cuestionario de Personalidad de Eysenck (Eysenck Personnality Questionnaire, EPQ- J y R; Eysenck \& Eysenck, 1975, 1997)}

Este instrumento permite evaluar tres dimensiones de la personalidad: Neuroticismo, Extraversión y Psicoticismo, más una medida adicional de sinceridad. Existen dos formas en función del rango de edad de aplicación: el EPQ-J (81 ítems) para niños y adolescentes de 8 a 14 años y el EPQ-R (83 ítems) para jóvenes a partir de 15 años y adultos. El formato de respuesta es dicotómico (Sí y No).

La consistencia interna para población española fue de .65 a .82 (EPQ-J) (Eysenck \& Seisdedos, 1978) y de .72 a .87 (EPQ-R) (Escorial \& Navas, 2007). La fiabilidad test-retest, para un período de seis meses en población británica fue de .61 a .88 (EPQ-J) (Eysenck \& Eysenck, 1975). La validez de constructo fue analizada mediante correlaciones con autoinformes de adaptación, inteligencia y depresión, hallándose índices aceptables (Eysenck \& Seisdedos, 1978). En este sentido, Inglés et al. (2001), sirviéndose en una muestra compuesta por 538 estudiantes españoles de 12 a 18 años, encontraron que las altas puntuaciones en neuroticismo y bajas en extroversión (introversión) eran predictores significativos de la fobia social, el miedo a hablar en público y las dificultades interpersonales en la adolescencia. En este estudio los valores de consistencia interna (Alpha de Cronbach) de las pruebas fueron .79 Extraversión, .68 Neuroticismo, .65 Psicoticismo (EPQ-J) y .83 Extraversión, .75 Neuroticismo y .73 Psicoticismo (EPQ-R).

\section{Procedimiento}

Se llevó a cabo una entrevista con los directores y psicopedagogos de los centros participantes para exponer los objetivos del estudio, describir los instrumentos, solicitar los permisos pertinentes y promover su colaboración. Posteriormente, se celebró una reunión con los padres para explicarles el estudio y solicitar el 
consentimiento activo e informado por escrito, autorizando a sus hijos a participar en la investigación. Las pruebas fueron contestadas de forma colectiva y anónima en el aula a finales del curso escolar. Los investigadores estuvieron presentes durante la administración de las pruebas para proporcionar ayuda si era necesaria y verificar la cumplimentación independiente por parte de los participantes.

\section{Análisis estadísticos}

La identificación de estudiantes con y sin ansiedad social se estableció a partir del punto de corte propuesto por Olivares et al. (2002). Así, la muestra general $(N=2022)$ se dividió en dos grupos: (a) sujetos sin ansiedad social: puntuaciones inferiores a $100\left(n_{l}=1778 ; 87.94 \%\right)$ y; (b) sujetos con ansiedad social: puntuaciones iguales o superiores a $100\left(n_{2}=244 ; 12.06 \%\right)$.

Para analizar las diferencias en personalidad entre estudiantes con baja y alta ansiedad social se aplicó la prueba $t$ de Student. Debido al elevado tamaño muestral del estudio, la prueba $t$ puede detectar erróneamente diferencias estadísticamente significativas. Por esta razón se incluye, además, el índice $d$ (diferencia media tipificada) propuesto por Cohen (1988), que permite valorar la magnitud o el tamaño del efecto de las diferencias encontradas. Su interpretación es sencilla: $0.20 \leq d \leq 0.50$ supone un tamaño del efecto pequeño, mientras que $0.51 \leq d \leq 0.79$ es moderado y $d \geq 0.80$ es grande.

Con el fin de examinar y cuantificar la capacidad predictiva entre las variables, se llevaron a cabo análisis de regresión logística binaria siguiendo el procedimiento de regresión por pasos hacia delante basado en el estadístico de Wald. Así, la ansiedad social y la personalidad fueron dicotomizadas cuando actuaban como variables criterio. Para la ansiedad social se mantuvo la misma dicotomización que para los análisis de diferencias de medias, mientras que las puntuaciones de cada subescala de la personalidad fueron dicotomizadas como: a) baja puntuación en rasgos de personalidad: puntuaciones iguales o inferiores al centil $25 \mathrm{y}$, b) alta puntuación en rasgos de personalidad: puntuaciones iguales $\mathrm{o}$ superiores al centil 75. Las puntuaciones de la personalidad se trataron de forma continua cuando esta actuaba como variable independiente.
El modelado logístico permite estimar la probabilidad de que ocurra un evento, suceso o resultado (e.g., alto neuroticismo) en presencia de uno o más predictores (e.g., alta ansiedad social). Esta probabilidad es estimada mediante el estadístico denominado odds ratio (OR). Si la OR es mayor que uno el aumento de la variable independiente lleva asociado un aumento en la ventaja del suceso. Por el contrario, si la OR es menor que uno el aumento de la variable independiente conlleva una disminución de la ventaja del suceso (De Maris, 2003).

\section{Resultados}

\section{Variables de personalidad de estudiantes con y sin alta ansiedad social}

A partir de la versión del cuestionario de personalidad administrado a los estudiantes, la muestra se dividió en dos submuestras: la primera, compuesta por los estudiantes más jóvenes $\left(1^{\circ} \mathrm{y}\right.$ $2^{a}$ ESO) a los que se administró la versión junior (EPQ-J) y, la segunda, compuesta por los alumnos de $3^{\circ}$ y $4^{\circ}$ ESO a los que se administró la versión revisada para adolescentes mayores de 15 años (EPQ-R). Se realizaron las pruebas $t$ de diferencias de medias para cada una de las submuestras.

La Tabla 1 recoge las puntuaciones medias de los rasgos de personalidad (extraversión, neuroticismo y psicoticismo), las diferencias estadísticamente significativas halladas entre estudiantes con y sin ansiedad social, así como el tamaño del efecto de las mismas.

Los estudiantes con alta ansiedad social presentaron puntuaciones medias significativamente más altas en el rasgo de neuroticismo o inestabilidad emocional $\mathrm{y}$ puntuaciones significativamente más bajas en extraversión, siendo la magnitud de dichas diferencias alta $(d=0.81)$ y baja $(d=0.46)$, respectivamente, en los estudiantes más jóvenes, y moderada ( $d=0.57$ y 0.73$)$ en el grupo de estudiantes de $3^{\circ}$ y $4^{\circ}$ ESO (Cohen, 1988). Por tanto, los estudiantes socialmente ansiosos se muestran más nerviosos, preocupados y tensos y menos sociables y activos que los estudiantes sin ansiedad social. 
Tabla 1. Diferencias de rasgos de personalidad entre estudiantes con y sin alta ansiedad social

\begin{tabular}{lccccc}
\hline & $\begin{array}{c}\text { Baja Ansiedad } \\
\text { Social }\end{array}$ & $\begin{array}{c}\text { Alta Ansiedad } \\
\text { social }\end{array}$ & \multicolumn{2}{c}{$\begin{array}{c}\text { Significación estadística y } \\
\text { magnitud de las diferencias }\end{array}$} \\
\cline { 2 - 6 } & $M(D T)$ & $M(D T)$ & $t$ & $p$ & $d$ \\
\hline EPQ-J $\left(1^{\circ}\right.$-2 $^{\circ}$ ESO) $(n=1097)$ & & & & \\
\hline Extraversión & $18.46(3.23)$ & $16.96(3.31)$ & 5.00 & .00 & 0.46 \\
Neuroticismo & $9.63(4.08)$ & $12.87(3.76)$ & -8.68 & .00 & -0.81 \\
Psicoticismo & $3.53(2.81)$ & $3.75(2.89)$ & -0.86 & n.s. & - \\
\hline EPQ-R (3⿻-4 ${ }^{\circ}$ ESO) $(n=925)$ & & & & .00 & 0.57 \\
\hline Extraversión & $14.39(3.28)$ & $12.48(3.73)$ & 5.64 & .00 & -0.73 \\
Neuroticismo & $11.28(4.93)$ & $14.86(4.50)$ & -7.22 & .00 & - \\
Psicoticismo & $6.14(3.47)$ & $6.18(3.32)$ & -0.11 & n.s. & \\
\hline
\end{tabular}

Nota. ESO=Educación Secundaria Obligatoria; n.s.=no significativo.

Tabla 2. Resultados derivados de la regresión logística binaria para la probabilidad de presentar altas puntuaciones en rasgos de personalidad

\begin{tabular}{|c|c|c|c|c|c|c|c|}
\hline & & B & E.T. & Wald & $p$ & OR & I.C. $95 \%$ \\
\hline & EPQ-J $\left(1^{\circ}-2^{\circ}\right.$ ESO $)$ & & & & & & \\
\hline \multirow[t]{2}{*}{ Extraversión } & Ansiedad social & -1.19 & .28 & 17.66 & .00 & 0.31 & $0.18-0.53$ \\
\hline & Constante & 0.20 & .09 & 5.20 & .02 & 1.22 & \\
\hline \multirow[t]{3}{*}{ Neuroticismo } & Ansiedad social & 2.22 & .35 & 40.74 & .00 & 9.17 & $4.65-18.12$ \\
\hline & Constante & -0.20 & .09 & 5.60 & .02 & 0.82 & \\
\hline & EPQ-R $\left(3^{\circ}-4^{\circ} \mathrm{ESO}\right)$ & & & & & & \\
\hline \multirow[t]{2}{*}{ Extraversión } & Ansiedad social & -1.41 & .30 & 21.87 & .00 & 0.24 & $0.14-0.44$ \\
\hline & Constante & 0.25 & .10 & 7.03 & .01 & 1.29 & \\
\hline Neuroticismo & Ansiedad social & 1.99 & .37 & 28.81 & .00 & 7.28 & $\begin{array}{l}3.53- \\
15.03\end{array}$ \\
\hline
\end{tabular}

Nota. $\mathrm{B}=$ coeficiente; E.T.=error estándar; $p=$ probabilidad; OR=odds ratio; I.C.=intervalo de confianza al $95 \%$.

\section{¿Es la alta ansiedad social un predictor significativo de la extraversión, el neuroticismo y el psicoticismo?}

Fue posible crear cuatro modelos predictivos para los rasgos de personalidad a través de la puntuación en ansiedad social (véase Tabla 2). El modelo predictivo para la extraversión de $1^{\circ}$ y $2^{\circ}$ ESO permite una estimación correcta del $57.1 \%$ de los casos $\left(\chi^{2}=19.74 ; p=.00\right)$ y el modelo predictivo para el neuroticismo permite una estimación correcta del $59.4 \%$ de los casos $\left(\chi^{2}=61.76 ; p=.00\right)$ entrando a formar parte de los modelos la variable ansiedad social. El valor de ajuste de los modelos ( $\mathrm{R}^{2}$ Nagelkerke) se situó en .04 para el modelo de extraversión y .12 para el de neuroticismo.

El modelo predictor de la extraversión en $3^{\circ}$ y $4^{\circ}$ ESO permite una estimación correcta de $58.9 \%$ de los casos $\left(\chi^{2}=25.44 ; p=.00\right)$ y el modelo predictivo para el neuroticismo permite una estimación correcta $57 \%$ de los casos $\left(\chi^{2}=41.09\right.$; $p=.00)$ quedando incluida en los modelos la variable ansiedad social. El valor de ajuste de los modelos ( $\mathrm{R}^{2}$ Nagelkerke) fue .07 para el modelo de extraversión y .10 para el de neuroticismo.
Las OR del modelos logísticos para $1^{\circ}$ y $2^{\circ}$ ESO informan que los estudiantes con alta ansiedad social presentan un $69 \%$ menos probabilidad de presentar altas puntuaciones en extraversión y un $817 \%$ más de probabilidad de presentar un marcado rasgo de neuroticismo. Por otra parte, las OR del modelos logísticos para $3^{\circ} \mathrm{y}$ $4^{\circ}$ ESO informan que los estudiantes con alta ansiedad social presentan un $76 \%$ menos de probabilidad de presentar altas puntuaciones en extraversión y $628 \%$ más de probabilidad de presentar altas puntuaciones en inestabilidad emocional.

\section{¿Son los tres rasgos de personalidad predictores significativos de la alta ansiedad social?}

A partir de la muestra analizada fue posible crear dos modelos logísticos para la predicción de ansiedad social a partir de los rasgos de personalidad extraversión, neuroticismo y psicoticismo (véase Tabla 3). El modelo predictivo para la ansiedad social en $1^{\circ}$ y $2^{\circ}$ ESO permite una estimación correcta del $88 \%$ de los casos $\left(\chi^{2}=84.61 ; p=.00\right)$, entrando a formar parte del modelo las variables extraversión y 
Tabla 3. Resultados derivados de la regresión logística binaria para la probabilidad de presentar alta ansiedad

\begin{tabular}{lccccccc}
\multicolumn{8}{c}{ social } \\
\hline & & B & E.T. & Wald & $p$ & OR & I.C. $95 \%$ \\
\hline \multirow{4}{*}{ Ansiedad } & EPQ-J $\left(1^{\circ}-2^{\circ}\right.$ ESO $)$ & & & & & & \\
social & Extraversión & -0.09 & .03 & 11.24 & .00 & 0.91 & $0.86-0.96$ \\
& Neuroticismo & 0.19 & .03 & 54.79 & .00 & 1.21 & $1.15-1.27$ \\
& Constante & -2.46 & .61 & 16.07 & .00 & 0.09 & \\
Ansiedad & EPQ-R $\left(3^{\circ}-4^{\circ}\right.$ ESO) & & & & & & \\
social & Extraversión & -0.16 & .03 & 29.40 & .00 & 0.85 & $0.80-0.90$ \\
& Neuroticismo & 0.16 & .02 & 46.23 & .00 & 1.17 & $1.12-1.23$ \\
& Constante & -1.96 & .49 & 16.09 & .00 & 0.14 & \\
\hline
\end{tabular}

Nota. $\mathrm{B}=$ coeficiente; E.T.=error estándar; $p=$ probabilidad; OR=odds ratio; I.C.=intervalo de confianza al $95 \%$.

y neuroticismo. El modelo predictor de la ansiedad social en $3^{\circ}$ y $4^{\circ}$ ESO permite una estimación correcta del $88.1 \%$ de los casos $\left(\chi^{2}=80.49 ; p=.00\right)$, quedando incluidas en el modelo las variables extraversión y neuroticismo. El valor de ajuste de los modelos se situó en $\mathrm{R}^{2}$ Nagelkerke de 0.14 para el modelo de $1^{\circ}$ y $2^{\circ}$ ESO y 0.16 para el de $3^{\circ}$ y $4^{\circ}$ ESO.

Las OR del modelo de $1^{\circ}$ y $2^{\circ}$ ESO indican que la probabilidad de presentar alta ansiedad social aumenta un $21 \%$ por cada unidad que incrementa la puntuación de la subescala Neuroticismo y disminuye un $9 \%$ por cada unidad que aumenta la puntuación de la subescala Extraversión. Las OR del modelo de $3^{\circ}$ y $4^{\circ}$ ESO revelan que la probabilidad de manifestar alta ansiedad social aumenta un $17 \%$ por cada unidad que incrementa la puntuación de la subescala Neuroticismo y disminuye un $15 \%$ por cada unidad que aumenta la puntuación de la subescala Extraversión.

\section{Discusión}

El presente estudio tuvo tres objetivos. En primer lugar, analizar las diferencias en variables de personalidad entre estudiantes españoles de ESO con y sin alta ansiedad social, en segundo lugar, determinar en qué medida la alta ansiedad social pronostica altas puntuaciones en neuroticismo, extraversión y psicoticismo, y en tercer lugar, examinar el papel explicativo que tienen los rasgos de personalidad sobre la ansiedad social.

De acuerdo con la primera, segunda y tercera hipótesis, este estudio comprobó que los estudiantes con alta ansiedad social, comparados a sus iguales sin alta ansiedad social, presentan un perfil de personalidad compuesto por altas puntuaciones en neuroticismo o inestabilidad emocional, bajas puntuaciones en extraversión y niveles similares en psicoticismo. Como señala Inglés et al. (2001), los rasgos de introversión y neuroticismo aparecen más en los adolescentes con dificultades interpersonales como la baja asertividad, problemas para hablar en público y relacionarse con el sexo opuesto, siendo los introvertidos los que tienen menos habilidades para mantener relaciones de amistad. Por tanto, los introvertidos e inestables emocionales, al presentar menores destrezas para relacionarse socialmente, pueden manifestar más aprehensión y nerviosismo ante las interacciones sociales, llevándoles a evitarlas o escapar de ellas. Esta evitación social, por un lado, refuerza negativamente el mantenimiento de la ansiedad social, ya que disminuye el malestar y la tensión, y por otro, impide el aprendizaje de nuevas habilidades sociales, ya que limita las oportunidades del adolescente a socializarse de un modo adecuado (La Greca \& López, 1998).

Igualmente, y coincidiendo con los hallazgos de estudios correlacionales y comparativos con muestras comunitarias de estudiantes (Mulder \& Aken, 2014; Zhao et al., 2014; Zhou et al., 2008) y muestras clínicas (Beidel et al., 2007), la alta ansiedad social predice una mayor probabilidad de obtener altas puntuaciones en neuroticismo y menor probabilidad de presentar un rasgo de personalidad extravertido. Por tanto, y según la cuarta hipótesis de estudio, los sujetos socialmente ansiosos presentan más riesgo de ser inestables emocionales e introvertidos.

Respecto a la tercera variable de personalidad evaluada (hipótesis cinco), coincidiendo con los hallazgos del estudio de Darwill et al. (1992) con estudiantes universitarios, los resultados indican 
que el rasgo de dureza o psicoticismo no resulta un factor predisponente para desarrollar ansiedad social en la adolescencia. Los sujetos con altos niveles de psicoticismo son impulsivos, antisociales, egocénticos, hostiles y poco empáticos, lo que les hace más propensos a desarrollar conductas de riesgo (Carvalho \& Novo, 2014) y psicopatologías de tipo externalizante como la esquizofrenia, transtorno antisocial de la personalidad o el abuso de drogas, pero no de problemáticas internalizantes como la ansiedad social.

Teniendo en cuenta los resultados, la sexta hipótesis fue confirmada, encontrando a los rasgos de extroversión y neuroticismo factores explicativos de la ansiedad social. Estos hallazgos coinciden con los obtenidos por Inglés et al. (2001) que reveló el poder explicativo de la introversión (o baja extraversión) sobre la ansiedad social. También están en consonancia con los resultados obtenidos por Calvete et al. (2016) que apuntan a las puntuaciones del neuroticismo y la extraversión como los indicadores sensibles para la predicción de la ansiedad social, siendo el último rasgo protector para el acoso entre iguales. De esta manera, el rasgo de neuroticismo en la adolescencia caracterizado por timidez, ansiedad, tensión, miedo, culpa, ideas irracionales, vergüenza, emotividad y preocupación, etc. es un factor de vulnerabilidad para desarrollar ansiedad social, mientras que la sociabilidad, asertividad, dominancia, osadía, espontaneidad de los jóvenes extravertidos les protege de manifestar inhibición conductual, y angustia o malestar ante situaciones sociales. Este fenómeno podría deberse a que las personas extravertidas y sociables tienen más oportunidades para poner en marcha habilidades de interacción social que pueden llevar a mostrarse más competentes y seguras cuando se relacionan con otras personas. Por el contrario, las personas con alto neuroticismo pueden llegar a evitar situaciones sociales por su bajo autoconcepto e inseguridad y preocupación por la evaluación de los demás y esto, les dificulte el aprendizaje de patrones de comportamiento social adaptativos.

Este estudio supera las limitaciones de estudios correlacionales realizados previamente, aportando nuevos datos sobre la relación predictiva entre ambas variables. Los hallazgos apuntan que la ansiedad social y el neuroticismo y extraversión son constructos que se relacionan significativa y bidireccionalmente, si bien, los resultados de las OR sugieren un mayor valor explicativo de la ansiedad social sobre el neuroticismo que sobre la extraversión. Estos resultados están en consonancia con otros hallazgos que señalan al neuroticismo como un factor de vulnerabilidad asociado a trastornos internalizantes como la ansiedad o la depresión, apareciendo la extraversión no tan directamente relacionada con los niveles de ansiedad y las disfunciones sociales (Calvete et al., 2016; Fumero \& Navarrete, 2015). Además, como señala Norton et al. (1997), presentar un menor interés hacia el contacto social, no es un factor tan determinante para la explicación de la ansiedad social como lo son los rasgos marcadamente ansiosos y aprehensivos.

Esta investigación no está exenta de limitaciones. En primer lugar, aunque el muestreo empleado garantiza la representatividad de la muestra reclutada respecto a la población diana, futuras investigaciones deberían confirmar si los resultados hallados en ESO difieren $\mathrm{o}$ se mantienen en otros niveles educativos. En segundo lugar, tampoco resultaría adecuado establecer generalizaciones de los hallazgos a estudiantes españoles de ESO diagnosticados con trastornos del aprendizaje o trastornos psicopatológicos, aspectos que pueden alterar claramente el comportamiento social y académico de los estudiantes. Además, teniendo en cuenta el principio de especificidad situacional que caracteriza el comportamiento social, difícilmente los resultados obtenidos podrían extrapolarse a estudiantes de Educación Secundaria de otras culturas y etnias. Por otra parte, en este estudio no se puede hablar de sujetos "con" o "sin" alta ansiedad social estrictamente, sino de sujetos con alta o baja ansiedad social en ausencia de un diagnóstico clínico. Por ello, el elevado porcentaje de sujetos que superan el punto de corte (12\%) es similar al de otros estudios tras la administración de escalas pero significativamente inferior cuando se cuenta con muestras clínicas (alrededor del 5\%) (Knappe, Sagagawa, \& Creswell, 2015). Además, el presente estudio se plantea conocer la capacidad predictiva de la alta ansiedad social sobre las 
variables de personalidad y viceversa, la capacidad predictiva de las variables de personalidad sobre la alta ansiedad social. Aunque es lógico pensar es que existe un efecto recíproco de ambas variables, futuras investigaciones podrían analizar esta cuestión elaborando dos modelos de ecuaciones estructurales para comprobar qué hipótesis es la más sostenible o, en cualquier caso, cuál es la fuerza de las asociaciones en ambos modelos. Además, sería aconsejable que futuros trabajos utilicen diseños longitudinales a fin de aportar datos más concluyentes respecto a las relaciones de influencia entre estas variables. Finalmente, la alta ansiedad social ha sido valorada únicamente mediante el punto de corte subclínico de un autoinforme. Por ello, consideramos que futuras investigaciones deberían incluir, además de una valoración de la ansiedad social autoinformada, la información derivada de entrevistas clínicas y de la aportada por otros agentes de información como padres y profesores.

A nivel práctico, el estudio de la ansiedad social en población infanto-juvenil podría aportar las claves necesarias para generar nuevas intervenciones preventivas eficaces en el ámbito escolar (Bernstein, Layne, Egan, \& Tennison, 2005), con el fin de identificar los casos de ansiedad social antes de que se cronifiquen, intervenir eficazmente sobre las áreas $\mathrm{y}$ funcionamiento afectado, así como, mejorar la calidad de vida y la integración grupal de los estudiantes inhibidos.

\section{Referencias}

Bados, A. (2001). Fobia social. Madrid: Síntesis. Barbaranelli, C., Caprara, G. V., \& Rabasca, A. (1998). Manuale del BFQ-C. Big Five Questionnaire Children. O.S. Organizzaaioni Speciali-Firenze.

Beidel, D. C., Turner, S. M., Young, B. J., Ammerman, R. T., Sallee, F. R., \& Crosby, L. (2007). Psychopathology of adolescent social phobia. Journal of Psychopathology and Behavioral Assessment, 29, 47-54. doi: 10.1007/s10862-006-9021-1

Bernstein, G. A., Bernat, D. H., Davis, A. M., \& Layne, A. E. (2008). School-based interventions for anxious children: 3-, 6-, and 12-month follow-ups. Journal of the American Academy of Child and Adolescent Psychiatry, 47, 1039-1047. doi: 10.1097/CHI.ob013e31817eecco

Bernstein, G. A., Layne A. E., Egan E. A., \& Tennison D. M. (2005). School-based interventions for anxious children. Journal of the American Academy of Child and Adolescent Psychiatry, 44(11), 1118-1127. doi: 10.1097/01.chi.0000177323.40005.a1

Blöte, A. K., Miers, A. C., Heyne, D. A., \& Westenberg, P. M. (2015). Social anxiety and the school environment of adolescents. In K. Ranta, A. M. La Greca, L. J. Garcia-Lopez, y M. Marttunen (Eds.,), Social Anxiety and Phobia in Adolescents: Development, manifestation and intervention strategies (pp. 183-199). Cham: Springer International Publishing.

Calvete, E., Orue, I., \& Gamez-Guadix, M. (2016). Do extraversion and neuroticism moderate the association between bullying victimization and internalizing symptoms? A threewave longitudinal study. Journal of School Psychology, 56, 111. doi: 10.1016/j.jsp.2016.02.003

Carvalho, R. G., \& Novo, R. F. (2014). Dimensões da personalidade e comportamentos de risco na adolescência: Um estudo com a versão portuguesa do MMPI-A. Revista Iberoamericana de Diagnóstico y Evaluación - e Avaliação Psicológica, 37, 203-222.

Cohen, J. (1988). Statistical power analysis for the behavioral sciences (2nd Ed.). Hillsdale, NJ: Erlbaum.

Costa, P. T., \& McCrae, R. R. (1999). NEO PI-R Manual. NEO PI-R, Inventario de Personalidad NEO Revisado. NEO-FFI, Inventario NEO reducido de Cinco Factores. Madrid: TEA Ediciones.

Darwill, T. J., Johnson, R. C., \& Danko, G. P. (1992). Personality correlates of public and private self-consciousness. Personality and Individual Differences, 13, 383-384.

Delgado, B., Inglés, C. J., \& García-Fernández, J. M. (2013). Social anxiety and self-concept in adolescence. Revista de Psicodidáctica 18(1), 179-195. doi:10.1387/RevPsicodidact.6411

Delgado, B., Inglés, C. J., \& García-Fernández, J. 
M. (2014). Ansiedad social y rendimiento académico en la adolescencia: Análisis de sus implicaciones teórico-prácticas. Estudios de Psicología, 35(1), 58-79. doi: 10.1080/02109395.2014.893649

De Maris, A. (2003). Logistic regression. En J.A. Schinka \& W. F. Velicer (Eds.), Research Methods in Psychology (pp. 509-532). New Jersey: John Wiley and Sons.

Ehrler, D. J., Evans, J. G., \& McGhee, R. L. (1999). Extending big-five theory into childhood: A preliminary investigation into the relationship between big-five personality traits and behavior problems in children. Psychology in the Schools, 36, 451-458. doi: 10.1002/(SICI)15206807(199911)36:6<451:: AID-PITS1>3.0.CO;2-E

Escorial, S., \& Navas, M. J. (2007). Analysis of the gender variable in the Eysenck Personality Questionnaire-Revised Scales using differential item functioning techniques. Educational and Psychological Measurement, 67, 990-1001. doi: 10.1177/001316440629910 8

Eysenck, H. J., \& Eysenck, S. B. G. (1964). Manual of the Eysenck Personality Inventory. London: University of London Press.

Eysenck, H. J., \& Eysenck, S. B. G. (1975). Manual of the Eysenck Personality Questionnaire - Junior (EPQ-J) and Adult (EPQ-A). Londres: Hodder \& Stoughton Educational.

Eysenck, H. J., \& Eysenck, S. B. G. (1997). Cuestionario revisado de personalidad de Eysenck $(E P Q-R)$. Madrid: TEA Ediciones.

Eysenck, S. B. G., \& Seisdedos, N. (1978). Estudio internacional de la personalidad. Revista de Psicología General y Aplicada, 33, 271-281.

Fumero, A., \& Navarrete, G. (2016). Personalidad y malestar psicológico: Aplicación de un Modelo de Redes Neuronales. Revista Iberoamericana de Diagnóstico y Evaluación - e Avaliação Psicológica, 41, 28-38.

García-López, L. J., De Los Reyes, A., \& Salvador, M. C. (2015). Assessment of social anxiety in adolescents. En K. Ranta, A. M. La Greca, L. J. García-López y M. Marttunen (Eds.,), Social Anxiety and Phobia in Adolescents: Development, manifestation and intervention strategies (pp. 121-150). Cham, Switzerland: Springer International Publishing.

Goldberg, L. R. (1999). A broad-bandwith, public-domain, personality in-ventory measuring the lower-level facets of several five-factor models. In I. Mervielde, I. Deary, F. De Fruyt y F. Ostendorf (Eds.), Personality psychology in Europe (Vol. 7., pp. 7-28). Tilburg, The Netherlands:Tilburg University Press.

Gren-Landell, M., Tillfors, M., Furmark, T., Bohlin, G., Andersson, G., \& Svedin, C.G. (2009). Social phobia in Swedish adolescents: Prevalence and gender differences. Social Psychiatry and Psychiatric Epidemiology, 44, 1-7. doi: 10.1007/s00127-008-0400-7

Halverson, C. F., Havill, V., Deal, J. E., Baker, S., Victor, J., \& Pavlopoulos, V. (2003). Personality structure as derived from parental ratings of free descriptions of children: The Inventory of Child Individual Differences. Journal of Personality, 71(6), 995-1026. doi: 10.1111/1467-6494.7106005

Inglés, C. J., Delgado, B. García-Fernández, J. M. Ruiz-Esteban, C., \& Díaz-Herrero. A. (2010). Tipos sociométricos y estilos de interacción social en una muestra de adolescentes españoles. Spanish Journal of Psychology, 13, 728-738. doi: 10.1017/S1138741600002390

Inglés, C. J., Hidalgo, M. D., \& Méndez, X. (2005). Interpersonal difficulties in adolescence: A new self-report measure. European Journal of Psychological Assessment, 20, 11-22. doi: 10.1027/10155759.21.1.11

Inglés, C. J., Méndez, F. X., \& Hidalgo, M. D. (2001). Dificultades interpersonales en la adolescencia: ¿Factor de riesgo de fobia social? Revista de Psicopatología y Psicología Clínica, 6, 91-104. doi: 10.5944/rppc.vol.6.num.2.2001.3907

Inglés, C. J., Piqueras, J. A., García-Fernández, J. M., García-López, L. J., Delgado, B., \& RuizEsteban, C. (2010). Diferencias de género y edad en respuestas cognitivas, psicofisiológicas y motoras de la ansiedad social en la adolescencia. Psicothema, 22, 376-381. 
John, O. P., \& Srivastava, S. (1999). The Big-Five trait taxonomy: History, measurement, and theoretical perspectives. In L. A. Pervin y O. P. John (Eds.), Handbook of personality: Theory and research (Vol. 2, pp. 102-138). New York: Guilford Press.

Knappe, S., Sagagawa, S., \& Creswell, C. (2015). Developmental epidemiology of social anxiety and social phobia in adolescents. En K. Ranta, A. M. La Greca, L. J. García-López y M. Marttunen (Eds.), Social Anxiety and Phobia in Adolescents: Development, manifestation and intervention strategies (pp. 39-70). Cham, Switzerland: Springer International Publishing.

La Greca, A. (1998). Manual for the Social Anxiety Scales for Children and Adolescents. Miami, FL: University of Miami.

La Greca, A. M., \& Lopez, N. (1998). Social anxiety among adolescents: Linkages with peer relations and friendships. Journal of Abnormal Child Psychology, 26(2), 83-94. doi: 10.1023/A:1022684520514

Merikangas, K. R., Avenenoli, S., Acharyya, S., Zhang, H., \& Angst, J. (2002). The spectrum of social phobia in the Zurich cohort study of young adults. Biological Psychiatry, 51, 8191. doi: 10.1016/S0006-3223(01)01309-9

Mulder, S. F., \& Aken, M. A. G. (2014). Socially anxious children at risk for victimization: The role of personality. Social Development, 23(4), 719-733.

Norton, G. R., Cox, B. J., Hewitt, P. L., \& McLeod, L. (1997). Personality factors associated with generalized and non generalized social anxiety. Personality Individual Differences, 22, 655-660. doi: 10.1016/S0191-8869(96)00243-7

Olivares, J., García-López, L. J., Hidalgo, M. D., La Greca, A. M., Turner, S., \& Beidel, D. (2002). A pilot study on normative data for two social anxiety measures: The Social Phobia and Anxiety Inventory and the Social Anxiety Scale for Adolescents. International Journal of Clinical and Health Psychology, 2, 464-476.

Olivares, J., García-López, L. J., Hidalgo, M. D., Turner, S., \& Beidel, D. (1999). The Social Phobia and Anxiety Inventory: Reliability and validity in an Adolescent Spanish population.
Journal of Psychopathology and Behavioral Assessment, 21, 67-78. doi: 10.1023/A:10228 64211676

Slobodskaya, H. R. (2007). The associations among the Big Five, Behavioural Inhibition and Behavioural Approach systems and child and adolescent adjustment in Russia. Personality and Individual Differences, 43(4), 913-924. doi: 10.1016/j.paid.2007.02.012

Turner, S. M., Beidel, D. C., Dancu, C. V., \& Stanley, M. A. (1989). And empirically derived inventory to measure social fears and anxiety: The Social Phobia and Anxiety Inventory. Psychological Assessment, 1, 3540. doi: 10.1037/1040-3590.1.1.35

Van Roy, B., Kristensen, H., Groholt, B., \& Clench-Aas, J. (2009). Prevalence and characteristics of significant social anxiety in children aged 8-13 years: A Norwegian crosssectional population study. Social Psychiatry and Psychiatric Epidemiology, 44(5), 407405. doi: 10.1007/s00127-008-0445-7

Woodward, L. J., \& Fergusson. D. M. (2001). Life course outcomes of young people with anxiety disorders in adolescence. The Journal of the American Academy of Child and Adolescent Psychiatry, 40, 1086-1093. doi: 10.1097/00004583-200109000-00018

Zhao, X., Zhang, Y., Chen, L., \& Zhou, R. (2014). Effect of personality traits on social anxiety in adolescents: The mediating effect of emotion regulation. Chinese Journal of Clinical Psychology, 22(6), 1057-1061.

Zhou, X., Xu, Q., Inglés, C. J., Hidalgo, M. D., \& La Greca, A. M. (2008). Reliability and validity of the Chinese version of the Social Anxiety Scale for Adolescents. Child Psychiatry and Human Development, 39, 185200. doi: 10.1007/s10578-007-0079-0 